\title{
Polarization angular spectra of Galactic synchrotron emission on arcminute scales
}

\author{
M. Tucci ${ }^{1,2,3}$, E. Carretti $^{4}$, S. Cecchini ${ }^{4}$, L. Nicastro ${ }^{5}$, R. Fabbri ${ }^{6}$, B.M. Gaensler ${ }^{7}$, J.M. \\ Dickey $^{10}$, N.M. McClure-Griffiths ${ }^{10,11}$
}

\begin{abstract}
We study the angular power spectra of the polarized component of the Galactic synchrotron emission in the 28- $\mathrm{deg}^{2}$ Test Region of the Southern Galactic Plane Survey at $1.4 \mathrm{GHz}$. These data were obtained by the Australia Telescope Compact Array and allow us to investigate angular power spectra down to arcminute scales. We find that, at this frequency, the polarization spectra for $E$ - and $B$-modes seem to be affected by Faraday rotation produced in compact foreground screens. A different behavior is shown by the angular spectrum of the polarized intensity $P I=$ $\sqrt{Q^{2}+U^{2}}$. This is well fitted by a power law $\left(C_{P I \ell} \propto \ell^{-\alpha_{P I}}\right)$ with slope $\sim 1.7$, which agrees with higher frequency results and can probably be more confidently extrapolated to the cosmological window.
\end{abstract}

Subject headings: Radio continuum: ISM; Cosmic microwave background; Polarization; Methods: statistical

\section{Introduction and main results}

In recent years the measurement of the Cosmic Microwave Background (CMB) polarization has become one of the major aims of a large number of planned experiments. Its detection is however a technological challenge: so far, we have only upper limits on the CMB polarization level [see Staggs et al. (1999) for a review and the recent measurements by PIQUE (Hedman et al. 2001)

\footnotetext{
${ }^{1}$ Dipartimento di Fisica G. Occhialini, Università degli Studi di Milano-Bicocca, Piazza della Scienza 3, I-20126 Milano, Italy.

${ }^{2}$ I.N.F.N., Via Celoria 16, I-20133 Milano, Italy.

${ }^{3}$ Instituto de Fisica de Cantabria, Avda. Los Castros s/n, 39005 Santander, Spain.

${ }^{4}$ I.A.S.F/C.N.R., Sezione di Bologna, Via Gobetti 101, I-40129 Bologna, Italy.

${ }^{5}$ I.A.S.F./C.N.R., Sezione di Palermo, Via Ugo la Malfa 153, I-90146 Palermo, Italy.

${ }^{6}$ Dipartimento di Fisica, Università di Firenze, Via Sansone 1, I-50019 Sesto Fiorentino, Italy.

${ }^{7}$ Harvard-Smithsonian Center for Astrophysics, Cambridge MA 02138, U.S.A.

${ }^{10}$ Bolton Fellow

${ }^{11}$ Australia Telescope National Facility, CSIRO, PO Box 76, Epping, NSW 1710, Australia.
}

and POLAR (Keating et al. 2001) experiments]. Since the CMB polarization signal is expected to be less than $10 \%$ of the temperature anisotropies, instrumental sensitivities of a few $\mu \mathrm{K}$ or less are required. These will be probably reached by the forthcoming experiments: three space missions, MAP (Wright 1999), Planck (De Zotti et al. 1999) and SPOrt (which is completely devoted to the study of sky polarized emissions; Carretti et al. 2002) will measure the polarization on nearly the full sky, while several ground-based or balloonborne experiments are planned to observe small sky areas with high spatial resolution [for instance, AMIBA (Kesteven et al. 2002), BOOMERanG 2K2 (Masi et al. 2002) and BaR-SPOrt (Zannoni et al. 2002); see De Zotti (2002) for a short review].

The possibility of extracting information on cosmological parameters from CMB experiments is strictly related to the computation of the angular power spectra (APS), which in the presence of Gaussian statistics give a complete statistical description of the CMB emission. Although for the temperature fluctuations the APS is easily defined through the ordinary spherical-harmonic expan- 
sion, for polarization we need two different components, the "electric" $(E)$ and "magnetic" $(B)$ modes, in order to describe the polarization intensity and orientation (Kamionkowski, Kosowsky \& Stebbins 1997, Zaldarriaga \& Seljak 1997). In section 2.2 we will discuss the definition of the polarization APS, with particular attention to the small scale limit.

The detection of the CMB polarization is constrained by the presence of foreground emissions. Different techniques have been worked out to separate the cosmological signal from the Galactic and extra-galactic emissions; all the methods exploit the differences in the frequency and spatial behaviors. For this reason, the analysis of the APS has become a common tool for studying the foregrounds: in fact, its knowledge allows us to estimate the foreground contamination to the $\mathrm{CMB}$ signal at different frequencies and angular scales (or equivalently, spherical-harmonic index $\ell$ ). For the total intensity emission, information on the spatial properties of foregrounds is limited only to a small interval of frequencies and angular scales (see, e.g., Tegmark et al. 2000), and it is completely unsatisfactory between 20 and $90 \mathrm{GHz}$. For the polarization, the situation is even worse because of the lack of high resolution surveys covering large areas.

In this paper we consider the synchrotron emission, which is intrinsically highly polarized and is expected to be the dominant foreground at low frequencies. Several authors have estimated the APS of the polarized synchrotron using data obtained with different resolutions and from limited sky regions at various latitudes (for a review of the up-to-date surveys, see Tucci et al. 2000). On small sky patches, Fourier analysis has been applied to the Stokes parameters $Q$ and $U$ (from which APS of the $E-, B$-modes can be computed) and to the polarized intensity $P I=\sqrt{Q^{2}+U^{2}}$ (Tucci et al. 2000; Tucci et al. 2001; Bruscoli et al. 2002). Scalar and spin-weighted harmonic expansions have been respectively used for PI (Baccigalupi et al. 2001; Giardino et al. 2002) and for $Q$ and $U$ (Giardino et al. 2002). These computations are not equivalent in an important respect. Fourier analysis, being adequate for small sky patches, necessarily provides local effective spectra which for highly non-Gaussian fields (such as the Galactic synchrotron distribution) may be widely different from the global angular spectra. In practice such local spectra may be more important than the global ones for the separation of Galactic foregrounds from CMB. Further, we note that the sum $C_{P \ell}=C_{E \ell}+C_{B \ell}$, which is the quantity usually considered in CMB analyses, and $C_{P I \ell}$ provide different information. This important point has been noticed in Tucci et al. (2002), and will be discussed in Section 2.2.

Here we observe that it helps to explain some discrepancies appearing in the literature. Estimates of the synchrotron polarization spectra were performed from the Parkes surveys of the Southern (Duncan et al. 1995, 1997, hereafter D97) and Northern (Duncan et al. 1999, hereafter D99) Galactic Plane. For both surveys, sampling more than half of the Galactic Equator, the APS are nearly independent of longitude, and could be modeled by power laws with slopes $\alpha_{E} \simeq \alpha_{B}=$ $1.4 \div 1.5$ and $\alpha_{P I}=1.6 \div 1.8$ in the $\ell$-range $100 \div 800$ [Tucci et al. 2000, 2001, Baccigalupi et al. 2001, Bruscoli et al. 2002, Giardino et al. 2002 (although the latter obtained $\alpha_{P I}=2.37 \pm 0.21$ in the $\ell$-range $40 \div 250$ from D97 data)]. Out of the Galactic Plane five patches are available at intermediate latitudes, $5^{\circ} \leq|b| \leq 20^{\circ}$, from the survey by Uyaniker et al. (1999). The APS vary significantly there, with slopes ranging from 1 to $\sim 2.5$ (Baccigalupi et al. 2001, Bruscoli et al. 2002). At latitudes far from the Galactic plane the only available information comes from the lowresolution survey of Brouw \& Spoelstra (1976), covering about $40 \%$ of the sky at five frequencies in the range $408 \div 1411 \mathrm{MHz}$. From this survey, Bruscoli et al. (2002) found values of $\alpha_{E, B, P I}$ between $1 \div 2$ at scales $\ell<100$, in agreement with the results from higher resolution data. Using the same survey, Baccigalupi et al. (2001) studied the $P I$ field and found steeper spectra, with $\alpha_{P I} \simeq 3$.

The present paper extends the analysis of the APS of the polarized Galactic synchrotron to arcminute scales $\left(\ell=10^{3} \div 10^{4}\right)$, i.e., to angular scales smaller than in previous works by nearly one order of magnitude. The study of the synchrotron contribution on these scales is relevant for $\mathrm{CMB}$ observations. In fact, the angular scales $300 \lesssim \ell<2000$ are expected to be those where CMB should exhibit the highest level of polarized signal. Moreover, at $\ell>3000$ non-linear effects on $\mathrm{CMB}$ become important, producing polarized 
signal stronger than the primary spectrum. These include the Vishniac, patchy reionization and kinetic Sunyaev-Zeldovich effects ( $\mathrm{Hu} 2000$, Liu et al. 2001).

We make use of high-resolution polarization data taken from a test region for the Southern Galactic Plane Survey (McClure-Griffiths et al. 2001, Gaensler et al. 2001, hereafter G01) consisting of $1.4-\mathrm{GHz}$ observations carried out with the Australia Telescope Compact Array (ATCA). We find that the $E$ and $B$ spectra can be well approximated by a power law at $600 \lesssim \ell \lesssim 6000$, with a steep slope $\alpha_{E, B} \simeq 2.7 \div 2.9$. Moreover, we compute the spectrum of the polarized intensity, $P I$ and find that it is remarkably different from the above spectra, following a power law with $\alpha_{P I} \simeq 1.7$ on the whole $\ell$-range. We compare the ATCA APS with the APS computed in the corresponding patch from the $2.4 \mathrm{GHz}$ Parkes survey. We find a noticeable agreement for $C_{P I, \ell}$, but not for $C_{E, B, \ell}$. The different slopes found in the APS are interpreted in section 2.4 as due to effects of Faraday rotation produced by foreground screens. No evidence is found for a contribution of extragalactic point sources.

The behaviors of synchrotron APS on arcminute scales at $\mathrm{GHz}$ frequencies may be interesting for information on Galactic structure that spectra contain. They can tell us, in fact, about both the magnetohydrodynamic turbulence in the emitting region and the electron density fluctuations in the intervening medium (shown by Faraday rotation). In this connection, the comparison between $1.4 \mathrm{GHz}$ and $2.4 \mathrm{GHz}$ data may be useful to separate the transverse structure of the magnetic field in the emitting region and the longitudinal field in the foreground screens. This point is open to future studies.

\section{Data analysis}

\subsection{The Test Region of the Southern Galactic Plane Survey (SGPS)}

The data we use were obtained with the Australia Telescope Compact Array (ATCA), an interferometer located near Narrabi, NSW, Australia and consisting of five movable $22-\mathrm{m}$ antennas on a $3-\mathrm{km}$ track (a sixth fixed antenna was not used in these observations). The data presented here were derived from observations in multiple array configurations (see McClure-Griffiths et al. 2001), resulting in virtually complete coverage visibilities between baselines of $31 \mathrm{~m}$ and $765 \mathrm{~m}$. Observations were made in the ATCA's multi-channel continuum mode, resulting in 9 spectral channels spread across a total bandwidth of $96 \mathrm{MHz}$, with a center frequency of $1384 \mathrm{MHz}$ (see G01 for details). There is significant Faraday rotation across the $96-\mathrm{MHz}$ bandwidth of these data, which would considerably complicate our analysis. Therefore in the work presented here, we have only utilized images from a single spectral channel corresponding to a center frequency of $1404 \mathrm{MHz}$. Faraday rotation across the $8-\mathrm{MHz}$ bandwidth of this channel is negligible. The ATCA observations covered a small portion of the sky, with Galactic coordinates $325.5<l<332.5,-0.5<b<3.5$. This area was a pilot survey for the recently completed Southern Galactic Plane Survey (SGPS), the analysis of which is in progress.

Maps of the Stokes parameters $Q$ and $U$ were derived from the interferometric visibility data using the techniques described by G01. $Q$ and $U$ images were smoothed with a gaussian beam of FWHM $87^{\prime \prime} \times 67^{\prime \prime}$ and reprojected into Galactic coordinates. The sensitivity of the images was $\sim 1.5$ mJy beam $^{-1}$, except in a strip of width 0.5 around the edges of the field for which the sensitivity was $\sim 3$ mJy beam $^{-1}$.

Interferometric observations are sensitive only to a limited range of spatial scales. The largest scale that can be detected is usually determined by the shortest antenna spacing. In the ATCA case this spacing is $31 \mathrm{~m}$, which corresponds to an angular scale of $\sim 25^{\prime}$. Although the mosaicing process employed here allows information on larger scales to be recovered (Ekers \& Rots 1979; Cornwell 1988), it does not do so with uniform sensitivity up to the largest scale sampled. Therefore, we here only consider angular power spectra to be reliable at $\ell \gtrsim 600$, corresponding to an angular scale of $\sim 20$ arcmin.

The distribution of linearly polarized intensity from the Test Region is shown in Fig. 1. The most prominent structure is a bright region which extends across the longitude range $327^{\circ}<l \lesssim 331^{\circ}$, at latitude $\sim+1^{\circ}$. A "spur" extends away from this region at $l \simeq 328.5$. At the edges of the survey two areas with very low polarized emission (the "voids" discussed by G01) are notice- 
able: the first of these is approximately centered in $\left(331^{\circ} .8,1.2\right)$, the second one between $327^{\circ} \lesssim$ $l<329.5$ at latitude $\sim 0^{\circ}$.

A wealth of small-scale structures are found in the diffuse polarized emission. As discussed in detail by G01, some of these structures are produced by a non-uniform distribution of magnetic fields in the emitting regions, while in other cases small-scale structures in $Q$ and $U$ have been induced along the line of sight as a result of Faraday rotation in compact clumps of ionized gas. Many sets of polarimetric observations have now identified this effect, and have attributed it to inhomogeneities in the interstellar medium in the form of clouds or filaments of sizes from $1 \mathrm{pc}$ to 100 pc (Wieringa et al. 1993; Gray et al. 1999; Haverkorn, Katgert \& de Bruyn 2000).

It is interesting to compare the image in Fig. 1 with the same area of the sky observed by the Parkes telescope at $2.4 \mathrm{GHz}$ and at the resolution of 10'.4 (see Fig. 6 in G01). We expect that polarized structures induced by Faraday rotation to be poorly correlated between $1.4 \mathrm{GHz}$ and $2.4 \mathrm{GHz}$, because of the strong dependence of Faraday rotation and depolarization on both frequency and angular resolution. On the other hand, we expect a good correspondence between the two data-sets for polarized structures which are intrinsic to the emitting sources. This is probably the case for the bright and extended emission at $327^{\circ}<l \lesssim 331^{\circ}$. Areas of fainter polarized emission seen at 1.4 $\mathrm{GHz}$ instead seem to be poorly correlated with the 2.4-GHz data, indicating the effects of foreground Faraday rotation in those regions.

\subsection{The polarization angular power spec- tra}

The CMB temperature anisotropies are usually expanded in spherical harmonics, $\Delta T / T(\hat{n})=$ $\sum_{\ell m} a_{\ell m} Y_{\ell m}(\hat{n})$. Assuming Gaussian statistics, all information in the CMB is contained in the angular power spectrum, defined as $C_{\ell}=\left\langle\left|a_{\ell m}\right|^{2}\right\rangle$, where \langle\rangle denotes an average over all possible realizations of the sky. The best estimator for the APS is

$$
C_{\ell}=\frac{1}{2 \ell+1} \sum_{m}\left|a_{\ell m}\right|^{2} .
$$

The definition of the APS for the CMB polarization is more complex. A linearly polarized wave is described by the two Stokes parameters $Q$ and $U$, which give us the polarized intensity, $P I=\sqrt{Q^{2}+U^{2}}$, and the polarization direction angle $\phi=0.5 \operatorname{atan}(U / Q)$. The value of $\phi$ depends obviously on the choice of the coordinate system; in particular, the $Q$ and $U$ parameters change if we rotate the coordinate system.

The standard approach to define rotationally invariant APS from $Q$ and $U$ is provided by Zaldarriaga \& Seljak (1997) (see also Kamionkowski, Kosowsky \& Stebbins 1997 for an alternative approach). They describe the polarization field in terms of two quantities scalar under rotation, $E(\hat{n})$ and $B(\hat{n})$ (in analogy with electric and magnetic fields' properties under parity transformation). The relation between $E, B$ and $Q, U$ is non-local. In fact, to construct scalars under rotation at point $\hat{n}$, we need to average the Stokes parameters around circles centered at $\hat{n}$ (Zaldarriaga 2001). From the harmonic expansions of the electric and magnetic modes one defines the polarization spectra $C_{E \ell}$ and $C_{B \ell}$.

Since the analysis presented in this paper considers only small patches of the sky, we can locally approximate the sphere as a plane and, hence, replace the spherical harmonic decomposition with a plane wave expansion (the standard Fourier transforms) (Seljak 1997). This allows us to considerably simplify the calculations.

In the flat sky limit, we can fix a common coordinate system and use this to define the Stokes parameters at every point $\vec{\theta}$ in the plane of the sky. In this way, we can directly compute the Fourier components of $Q$ and $U$,

$$
\begin{aligned}
Q(\vec{\ell}) & =\int \mathrm{d} \vec{\theta} e^{-i \vec{\ell} \cdot \vec{\theta}} Q(\vec{\theta}) \\
& =\int \mathrm{d} \vec{\theta} e^{-i \vec{\ell} \cdot \vec{\theta}} P I(\vec{\theta}) \cos \left(2 \phi_{\vec{\theta}}\right) \\
U(\vec{\ell}) & =\int \mathrm{d} \vec{\theta} e^{-i \vec{\ell} \cdot \vec{\theta}} U(\vec{\theta}) \\
& =\int \mathrm{d} \vec{\theta} e^{-i \vec{\ell} \cdot \vec{\theta}} P I(\vec{\theta}) \sin \left(2 \phi_{\vec{\theta}}\right)
\end{aligned}
$$

where $\phi_{\vec{\theta}}$ is the angle of which the common axis must be rotated in order to have $U=0$ in the point $\vec{\theta}$. Using the Fourier transforms one can estimate the polarization power spectrum through:

$$
C_{X \ell}=\frac{1}{2 \pi} \int \mathrm{d}^{2} \ell^{\prime} X(\vec{\ell}) X^{*}(\vec{\ell}) \delta\left(\ell-\ell^{\prime}\right)
$$


with $X=Q, U$.

In the small scale limit the relation between electric and magnetic modes and the $Q, U$ Stokes parameters assumes a very simple expression, consisting of a rotation in the $\ell$-space:

$$
\begin{aligned}
& E(\vec{\ell})=Q(\vec{\ell}) \cos \left(2 \phi_{\vec{\ell}}\right)+U(\vec{\ell}) \sin \left(2 \phi_{\vec{\ell}}\right) \\
& B(\vec{\ell})=-Q(\vec{\ell}) \sin \left(2 \phi_{\vec{\ell}}\right)+U(\vec{\ell}) \cos \left(2 \phi_{\vec{\ell}}\right) .
\end{aligned}
$$

The $C_{E \ell}$ and $C_{B \ell}$ spectra are then computed using eq. 4 .

The same expressions used to define the CMB polarization spectra can be safely applied to foreground components and, in particular, to the synchrotron emission. However, we would like to stress some differences. First of all, the spatial distribution of synchrotron radiation, both in the total intensity and in its polarized components, exhibits strong non-Gaussian features and may depend on the sky position. For instance, the Galactic plane, the North Galactic Spur and discrete sources generally present an emission level that is much higher than the other parts of the sky. Now, considering the Test Region, Fig. 1 shows, together with an extended and bright region, areas with extremely low intensity levels. Such strong variations in the polarization intensity are due not only to the different intrinsic level of polarization, which we expect to be more uniform on the Galactic plane, but also reflect the different importance of the depolarizing mechanisms that occur inside the emitting regions or along the line of sight. Moreover, the polarized emission shows a weaker dependence with respect to $b$ than the total intensity. So, the polarization APS computed from this survey does not describe only the intrinsic spatial distribution of polarized emission and can locally vary according to the area considered. However, when we consider patches of the sky that are large with respect to the dimensions of the observed structures, the angular spectrum is expected to adequately describe the average spatial properties of the emission. In our analysis, we consider also several small patches $\left(1^{\circ} \times 1^{\circ}\right)$ in order to study how the spectrum shape changes among regions with faint and bright polarized emission.

Differences between CMB and foregrounds concern also the $B$-mode. For cosmological signals induced by scalar perturbations this quantity vanishes. It arises only in presence of vector or ten- sor perturbations and in any case its intensity level is smaller than the $E$-mode one (Zaldarriaga \& Seljak 1997). On the contrary, the synchrotron emission contributes, on average, with the same amount to both quantities and their spectra present a similar shape (Seljak 1997).

In order to estimate the APS from observational data, we need to consider in the above expression (4) the characteristics of the instrument. If $X(\vec{\theta})$ is the quantity measured by ATCA experiment on a square grid of $N$ pixels covering a solid angle $\Omega$, in the small scale limit the estimator of the angular power spectrum can be computed as

$$
C_{X \ell}=\frac{\Omega}{N_{\ell}} \sum_{\vec{\ell}} X(\vec{\ell}) X^{*}(\vec{\ell}) b_{\vec{\ell}}^{-2}-w^{-1},
$$

where $X(\vec{\ell})=N^{-1} \sum e^{-i \vec{\ell} \cdot \vec{\theta}} X(\vec{\theta})$ is the discrete Fourier transform of the data and corresponds to the quantities $Q, U, E, B$. The sums are performed over the $N_{\ell}$ independent modes with wavevector magnitude around $\ell$. In the present case the term $b_{\vec{\ell}}$ takes into account that the ATCA images were subsequently smoothed with an elliptical Gaussian window function of FWHM $\theta_{a} \times \theta_{b}=87^{\prime \prime} \times 67^{\prime \prime}$. The expression in Fourier space is

$$
b_{\vec{\ell}}=\exp \left\{-\frac{1}{2} \vec{\ell} \cdot \mathbf{M} \cdot \vec{\ell}\right\},
$$

where the matrix $\mathbf{M}$ embodies the beam properties. We checked that adopting a circular beam with $\theta_{\text {ave }}=77^{\prime \prime}$ would make very little difference. The contribution of the noise, which is smoothed by the window function $b_{\vec{\ell}}$, is subtracted by means of the factor $w^{-1}=\Omega_{b} \sigma^{2}$, i.e. the pixelindependent measure of noise ( $\sigma$ is the rms noise amplitude, and $\left.\Omega_{b}=\theta_{a} \times \theta_{b}\right)$.

Together with the electric and magnetic modes, in the following analysis we consider the APS also for the polarized intensity, $P I=\sqrt{Q^{2}+U^{2}}$. If $Q$ and $U$ are two Gaussian quantities, $P I$ will have a Ricean distribution and its standard deviation will be $\sigma_{P I}=0.66 \sigma_{Q, U}$. The polarized intensity is a scalar quantity and can be expanded in ordinary spherical harmonics; its power spectrum $C_{P I \ell}$ can be, therefore, defined in a way equivalent to the CMB temperature fluctuations spectrum. In the small scale limit, an expression equivalent to equation (6) can be used to compute $C_{P I \ell}$. In this case, 
the contribution of noise $\left(w_{P I}^{-1}\right)$ will be lower than those of $E$ and $B$ by a factor $\sim 0.43$. The function $b_{\vec{\ell}}$ is not exactly the antenna beam used for $Q$ and $U$, however this can be considered a good approximation for $b_{\vec{\ell}}$. The differences with the actual smoothing function become meaningful only at angular scales very close to the telescope resolution (they are responsible for the increasing of $C_{P I \ell}$ that we find at $\ell>8000$; see figures).

The $C_{P I \ell}$ spectrum must not be confused with the polarization power spectrum $C_{P \ell}$ defined in Seljak (1997), where $C_{P \ell}=C_{Q \ell}+C_{U \ell}=C_{E \ell}+$ $C_{B \ell}$, and clearly has a different physical meaning with respect to $C_{P I \ell}$. The latter, unlike the $E$ - and $B$-modes, does not provide a complete description of the polarization field because it is related only to the intensity of the polarization without giving any information on its position angle. Only if the polarization angle is uniform inside all the survey area, the equality $C_{P I \ell}=C_{P \ell}$ is ensured, while in general $C_{P I \ell}$ and $C_{P \ell}$ are expected to show different behaviors. This is surely the case for CMB polarization, as shown in Fig. 2 . There we report the $E$-mode (solid line) and PI (dashed line) spectra resulting from Fourier analysis on $\mathrm{CMB}$ simulated maps. We chose a standard cosmological model with only scalar perturbations, where therefore $C_{E \ell}=C_{P \ell}$, and simulation boxes of $10^{\circ} \times 10^{\circ}$.

As discussed in the Section 1, according to previous analysis of the synchrotron maps by Bruscoli et al. (2002), the polarized intensity APS has a slightly faster decrease than the $E$ and $B$-mode APS at $\ell \leq 800$. This is not surprising because we expect that the $E$ and $B$ modes vary more rapidly than $P I$, due to changes both in the intensity and in the angle of polarization.

\subsection{Results}

We study the APS of the synchrotron polarization in several square patches of the survey with different dimensions. Because of the limited sky area considered, the Fourier approach is suitable; after computing the Fourier components of the data, $Q(\vec{\ell}), U(\vec{\ell})$ and $P I(\vec{\ell})$, equation 6 is applied to estimate the spectra for the modes $E, B$ and $P I$ (the spectra for $Q$ and $U$ are redundant, as shown in the previous section). A standard least-squares method was used to fit each curve to a power law, $C_{X \ell}=A_{X} \ell^{-\alpha_{X}}$, in suitable $\ell$-ranges (see Tucci et al. 2000).

Fig. 1 shows the areas of the survey where the power spectra are computed: a $4^{\circ} \times 4^{\circ}$ box centered in $(l, b)=\left(329^{\circ}, 1^{\circ} .5\right)$, which is the largest square area that can be extracted from the survey; two $3^{\circ} \times 3^{\circ}$ boxes covering nearly all the highsensitivity part of survey; and six $1^{\circ} \times 1^{\circ}$ boxes, in order to sample regions with different features in polarization.

In the Test Region, 21 compact sources showing linear polarization were identified (see Table 1 in G01). Some of these can be readily seen in Fig. 1. We tried to choose the $1^{\circ} \times 1^{\circ}$ boxes in regions where the number of compact sources is not more than one. Also for the larger boxes we can fairly suppose that the number of compact sources is low enough not to affect the estimate of the power spectra.

First of all, let us discuss the results for $C_{E \ell}$ and $C_{B \ell}$ in the $4^{\circ} \times 4^{\circ}$ area. In Fig. 3 we report only $C_{E \ell}$ (solid line); the results for $B$-mode are similar to those for $E$-mode. In the range $600<\ell \leq 6000$ such spectra are well approximated by a power law with $\alpha_{E} \simeq \alpha_{B} \simeq 2.7 \div 2.9$ (see Table 1 ). We compare the $E$-mode spectrum to that resulting from the observations in the same sky area by the Parkes telescope (D97). In the plot the Parkes spectrum has been scaled to the ATCA frequency, assuming the frequency spectrum of synchrotron emission $T_{\text {syn }} \propto \nu^{\beta}$ with spectral index $\beta=-2.5$ and -3 (dashed lines). We compute the best fit for $100 \leq \ell \leq 800$ and find a power law index of $\alpha_{E}=1.78 \pm 0.18$. This value is significantly lower than the best-fit $\alpha_{E}$ found from the ATCA survey, i.e. $2.85 \pm 0.07$ (dotted line). Moreover, the amplitudes of the Parkes and ATCA spectra show a difference of nearly one order of magnitude at scales $600 \lesssim \ell \lesssim 1000$. In this range, corresponding to angular dimensions between $20^{\prime}$ and $10^{\prime}$, both Parkes and ATCA are able to detect polarization signals. This gap, therefore, must be related to the different frequency at which the observations were made and cannot be attributed to our choice for the synchrotron spectral index. In fact, to remove the discrepancy one requires a spectral index of $-5 \div-4$, i.e., values too far from the average estimate between these frequencies $(\beta=-2.8$, see Platania et al. 1998), even in a very peculiar region.

Quite different results come from the analysis 
of the polarized intensity spectrum. For the same $4^{\circ} \times 4^{\circ}$ survey patch (see Fig. 4 ) the $C_{P I \ell}$ is well approximated by a power law with $\alpha_{P I}=1.66 \pm$ 0.07 (the dotted line is the best-fit in $600<\ell \leq$ 6000). The $P I$ spectrum shows a much flatter shape than $C_{E, B \ell}$, and looks like the extension at high $\ell$ of the curves obtained from $2.4 \mathrm{GHz}$ Parkes data.

Similar results are obtained from the analysis of smaller areas. In Fig. 5 we directly compare the electric and magnetic spectra with $C_{P I \ell}$, computed on two $3^{\circ} \times 3^{\circ}$ regions that sample all the low-noise part of the survey. Table 1 reports the best-fit parameters of the curves. These plots make evident how the $E$ - and $B$-mode spectra have a similar shape to each other but separate from $C_{P I \ell}$ as they move toward low $\ell$.

In Fig. 6 we plot $C_{E \ell}$ and $C_{P I \ell}$ obtained in $1^{\circ} \times 1^{\circ}$ regions, chosen between high- and lowpolarized emission areas (see the rms values of the polarized intensity $P I_{\text {rms }}$ in Table 2). We use letters $a$ through $f$ to label six areas. The best-fit parameters for the curves are reported in Table 2 . We found that the slope of both $E-, B$-mode and $P I$ spectra in $1^{\circ} \times 1^{\circ}$ regions are quite independent of the area considered, with only small fluctuations around the values found in the bigger regions. For example, the spectral slope does not significantly differ between the regions $a-b$, that are within the brightest area of the Test Region, and $e-f$, where only faint polarized emission is observed. The only exception is the $d$ case, which corresponds to a peculiar region inside which 1.4 and $2.4 \mathrm{GHz}$ data show anti-correlation, probably produced by internal Faraday depolarization occurring at $1.4 \mathrm{GHz}$. In this area $C_{E, B \ell}$ and $C_{P I \ell}$ are much steeper than in the other regions, indicating that internal depolarization erases intrinsic small-scale structures.

\subsection{ISM structures, Faraday screens and point sources}

The strong differences between $C_{P I \ell}$ and $C_{E, B \ell}$ found at $1.4 \mathrm{GHz}$ but not at $2.4 \mathrm{GHz}$, as well as the amplitude gap in $E$-mode APS shown in Fig. 3 can be interpreted if we assume that polarization structures are not intrinsic to the emitting regions. As already discussed by G01 and in section 2.1 , an alternative mechanism to produce smallscale structures is the Faraday rotation along the line of sight due to foreground screens. In detail, if a smooth polarized background emission passes through an interstellar medium (ISM) with spatial variations in the rotation measure, the linearly polarized radiation is affected by a Faraday rotation of the polarization angle that varies spatially with the line of sight. Hence, if the angular dimension of these ISM structures are sampled by the interferometer, the Faraday rotation will induce detectable variations in $Q$ and $U$, and the $E$ and $B$ spectra will gain extra power on these scales. Visual inspection of Fig. 5 of G01, reporting the polarization angle, apparently shows many sharp-edge structures with size of the order of $10^{\prime}$, supporting our interpretation.

The situation is quite different for the polarized intensity spectrum, $C_{P I \ell}$. The $P I$ value is not changed by a rotation of the polarization angle, but it could be affected by depolarization effects that the rotation induces. Some areas, where Faraday rotation may be the cause of a strong reduction of the polarized intensity, are found by G01: for instance, the existence of two large regions devoid of polarized emission is explained by beam depolarization due to large RM variations on small scales. Their position is, however, at the edges of the survey and their contribution to the APS of our selected regions can be considered negligible. Internal Faraday depolarization could be the cause of the anti-correlation of the polarized emission between 1.4- $\mathrm{GHz}$ and 2.4- $\mathrm{GHz}$ data, observed in the "spur" region (labeled as $d$, cfr. Section 2.3). However, apart from this peculiar region, the low RM values measured in the rest of the ATCA survey suggest that Faraday depolarization should not affect our estimates of the polarized intensity spectrum, especially in the large boxes. This is confirmed by $\alpha_{P I}$ values found in $1^{\circ} \times 1^{\circ}$ regions, which result weakly dependent on the area considered and on the magnitude of RM measured there (see Table 2). So, in the absence of depolarization, the Faraday rotation changes only the polarization angle without affecting the intensity and consequently $C_{P I \ell}$.

The effects of Faraday screens on the polarization emission have been discussed by previous authors. Wieringa et al. (1993) were the first to notice that the polarized component of the galactic background at $325 \mathrm{MHz}$ is characterized by a patchy pattern on arcminute scales. They 
detected features like narrow filaments of $5^{\prime}-10^{\prime}$ width and "clouds" with angular sizes of some tens of arcminutes, across which there are only small changes in the polarization angle. These structures were found at different latitudes and were just interpreted in terms of Faraday modulation by foreground ionized gas clumps. More recent interferometric observations (Duncan et al. 1998; Gray et al. 1999; Haverkorn et al. 2000) confirmed these features in polarization even at $1.4 \mathrm{GHz}$.

In Faraday-induced polarization structures observed by Wieringa et al. (1993), excess RMs from compact screens were measured with typical magnitude $\sim 5 \mathrm{rad} \mathrm{m}^{-2}$, corresponding to $\Delta \phi \sim 13^{\circ}$ at $1.4 \mathrm{GHz}$. Although small, these RM values suffice to produce structures which are detectable by the ATCA. Following the appendix in G01, if uniformly polarized emission with intensity $P_{0}$ passes through a compact cloud of rotation measure $R_{c}$, the interferometer detects polarized emission in the direction of the cloud with apparent intensity

$$
P_{\text {det }}=2 P_{0} \sin \left(R_{c} \lambda^{2}\right),
$$

and measures the RM value of the cloud as $R_{c} / 2$. Then, using $R_{c}=10 \mathrm{rad} \mathrm{m}^{-2}$, at $1.4 \mathrm{GHz}$, the polarization induced by a compact cloud will be close to $100 \%$ of the background component. At $2.4 \mathrm{GHz}$ (the frequency of the D97 survey) this percentage is reduced by about a factor of three, without considering the depolarization effects due to the lower resolution.

The above discussion assumes that none of the features of angular spectra should be attributed to extragalactic point sources. This is consistent with estimates: from Tegmark and Efstathiou (1996), using a VLA sample of $1.5 \mathrm{GHz}$ sources, flat intensity spectra $C_{I \ell}^{\mathrm{PS}}=(3 \div 13) \times 10^{-8} \mathrm{~K}^{2}$ are derived for limiting fluxes in the range $(0.1 \div 1)$ Jy. From Toffolatti et al. (1998) considering an evolution model for galaxies, for the same limiting fluxes and the (frequency) spectral index in the range $-(2 \div 2.3)$ we get $C_{I \ell}^{\mathrm{PS}}=(0.2 \div 6.6) \times 10^{-8} \mathrm{~K}^{2}$. A more conservative upper limit can be derived from our own analysis of intensity spectra in U99 (Tucci et al. 2001, Bruscoli et al. 2002). Some patches exhibit very flat spectra there, $\alpha_{I} \simeq 0$; assuming that they are dominated from point sources, we get the limit $C_{I \ell}^{\mathrm{PS}}<5 \times 10^{-7} \mathrm{~K}^{2}$. From this number, adopting a radio-source polarization degree of $5 \%$ [in agreement with De Zotti et al. (2000)] we get $C_{X \ell}^{\mathrm{PS}}<1.3 \times 10^{-9} \mathrm{~K}^{2}$ for $X=P, P I$, and we conclude that the contribution of point sources should be negligible in the whole range $\ell \leq 6000$. This is certainly consistent with the simple behavior of the angular spectra that we find.

\section{Discussion}

In this paper, for the first time, we extend the study of the angular power spectrum for the polarized component of the Galactic synchrotron emission to arcminute scales, i.e. up to $\ell \sim 10^{4}$. To reach such scales we needed high-resolution data, which were provided by the ATCA observations of a small patch of the Galactic Plane at $1.4 \mathrm{GHz}$.

In the paper we compute the polarization spectra for "electric" and "magnetic" modes, plus the spectrum for the polarized intensity. We find that, in the range $600 \leq \ell \leq 6000$, both $C_{E \ell}$ and $C_{B \ell}$ can be well approximated by power laws with slopes $\alpha_{E} \simeq \alpha_{B} \sim 2.7 \div 2.9$. Such spectra are significantly steeper than those arising at $\ell \leq 800$ from low-resolution data. Moreover, their amplitude, if compared to the spectra obtained by the Parkes telescope in the same sky area, turn out to be higher by nearly one order of magnitude at angular scales between $20^{\prime}$ and $10^{\prime}$. These peculiar behaviors are well interpreted as due to the small-scale modulation of a relatively uniform polarized background by Faraday rotation along the line of sight. On the contrary, we believe that our estimates of $C_{P I \ell}$, whose slope $\left(\alpha_{P I} \sim 1.7\right)$ is in agreement with D97 data at $2.4 \mathrm{GHz}$, are not affected by Faraday effects and fairly describe the intrinsic spatial distribution of the polarized emission.

An interesting point, which arises from our analysis, regards the distinctive meaning of $C_{P I \ell}$ with respect to $C_{E, B \ell}$. As we have discussed in section $2.2, P I$ is a scalar quantity and refers only to the intensity of the polarization without any information on its direction. We then expect that the APS for $E$ - and $B$-modes, that provide a complete description of the polarization field, do not have the same shape as the $P I$ spectrum. The differences should be greater when the direction of polarization changes very rapidly. Deviations between $C_{E \ell}$ and $C_{P I \ell}$ are found in the CMB (see the results of the simulations in Fig. 2): these are not unexpected, because of the geometry of the po- 
larization angle in the $E$-mode spots. In the case of synchrotron emission, the polarization direction for the diffuse component is quite smooth on large scales following the Galactic magnetic field. From low-resolution surveys the estimates of $C_{E, B \ell}$ and $C_{P I \ell}$ give only moderate differences in the spectral shape (see Bruscoli et al. 2002). However, when small scales are considered, fluctuations in magnetic fields, discrete sources and also Faraday effects contribute to amplify the variations in the polarization spectra, as the present results highlight.

The extrapolation of our results for $C_{E \ell}$ and $C_{B \ell}$ to higher frequencies should not be regarded as a trivial matter, since Faraday effects are substantial at $1.4 \mathrm{GHz}$ while they become negligible at a few tens of GHz. The electric and magnetic spectra are strongly affected by the Faraday rotation along the line of sight, showing a steep slope $\left(\alpha_{E, B} \sim 2.8\right)$. The polarized intensity spectrum, instead, can be more reliably extrapolated to the "cosmological" frequencies, because, as discussed in section 2.4, it is not affected by Faraday rotation, except in severe cases when significant depolarization is occurring. The power index $\alpha_{P I}$ is less than 2 independent of the region analysed, with a value of $1.66 \pm 0.05$ in the $4^{\circ} \times 4^{\circ}$ box.

We have seen that analyses on the synchrotron polarization spectrum in the literature indicate a moderate slope $\left(\alpha_{X} \lesssim 2\right.$ with $\left.X=E, B, P I\right)$ on angular scales $\ell<10^{3}$. Now ruling out a significant contribution from point sources, we confirm this result also at $\ell \lesssim 10^{4}$ for $C_{P I \ell}$, and we show that synchrotron emission is rather rich in small scale structures. Hence, contrary to what is usually assumed, it might be a relevant contaminant in $\mathrm{CMB}$ polarization measurements at very small scales. It remains to be seen if the APS as deduced by ATCA observations of the Galactic plane is a common feature in regions at high galactic latitudes. In general we expect the polarized synchrotron emission to be fainter in regions far out of the Galactic plane, except in very bright areas. In one such region Bruscoli et al. (2002) estimate $C_{X \ell}$ for $\ell<100$, finding a spectrum behavior consistent with those of the Galactic plane $\left(1<\alpha_{X}<2\right)$. Even if very bright regions are not typical at high latitudes, these play an important role in the process of foreground subtraction in $\mathrm{CMB}$ experiments; this is why we have to put great care into the study of the APS of these regions.

We thank the whole SPOrt collaboration team and, in particular, M.T. wishes to thank S. Bonometto for encouragement and useful discussions. This work is supported by the Italian Space Agency (ASI). M.T. acknowledges the financial support provided through the European Community's Human Potential Programme under contract HPRN-CT-2000-00124, CMBNET. The Australia Telescope is funded by the Commonwealth of Australia for operation as a National Facility managed by CSIRO. B.M.G. acknowledges the support of a Clay Fellowship awarded by Harvard-Smithsonian Center for Astrophysics. N.M.Mc-G. and J.M.D. acknowledge the support of NSF grant AST-9732695 to the University of Minnesota.

\section{REFERENCES}

Baccigalupi, C., Burigana, C., Perrotta, F., De Zotti, G., La Porta, L., Maino, D., Maris, M., \& Paladini, R., 2001, A\&A, 372, 8.

Brouw, W.N., \& Spoelstra, T.A., 1976, A\&AS, 26, 129.

Bruscoli, M., Tucci, M., Natale, V., Carretti, E., Fabbri, R., Sbarra, C., \& Cortiglioni, S., 2002, NewA, 7, 171.

Carretti, E., et al., 2002, in: Astrophysical Polarized Backgrounds, Cecchini, S., Cortiglioni, S., Sault, R., \& Sbarra, C. (eds.), AIP Conference Proc., 609, 109; and SPOrt home page: http://sport.tesre.bo.cnr.it/.

Cornwell, T.J., 1988, A\&A, 202, 316.

De Zotti, G., Gruppioni, C., Ciliegi, P., Burigana, C., \& Danese, L., 1999, NewA, 4, 481.

De Zotti, G., et al., 1999, in: 3 K Cosmology, Maiani, L., Melchiorri, F. \& Vittorio, N. (eds.), AIP Conference Proc. 476, 204; and PLANCK home page: http://astro.estec.esa.nl/SAgeneral/Projects/Planck.

De Zotti, G., et al., 2002, in: Astrophysical Polarized Backgrounds, Cecchini, S., Cortiglioni, S., Sault, R., \& Sbarra, C. (eds.), AIP Conf. Proc., 609, 295. 
Duncan, A.R., Stewart, R.T., Haynes, R.F., \& Jones, K.L., 1995, MNRAS, 277, 36.

Duncan, A.R., Haynes, R.F., Jones, K.L., \& Stewart, R.T., 1997, MNRAS, 291, 279 (D97).

Duncan, A.R., Haynes, R.F., Reich, P., Reich, W. \& Gray, A.D., 1998, MNRAS, 299, 942.

Duncan, A.R., Reich, P., Reich, W. \& Fürst, E., 1999, A\&A, 350, 447 (D99).

Ekers, R.D., \& Rots, A.H., 1979, in Image Formation from Coherence Functions in Astronomy, ed. C. van Schooneveld, (Dordrecht: Reidel), p.61.

Gaensler, B.M., Dickey, J.M., McClure-Griffiths, N.M., Green, A.J., Wieringa, M.H., \& Haynes, R.F., 2001, ApJ, 549, 959 (G01).

Giardino, G., Banday, A.J., Górski, K.M., Bennet, K., Jonas, J.L., \& Tauber, J., 2002, Astronomy \& Astrophysics, in press; astro-ph/0202520.

Gray, A.D., et al., 1999, ApJ, 221.

Haverkorn, M., Katgert, P., \& de Bruyn, A.G., 2000, A\& A, 356, L13.

Hedman, M.M., Barkats, D., Gundersen, J.O., Staggs, S.T., \& Winstein, B., 2001, ApJ, 548, L111.

Hu, W., 2000, ApJ, 529, 12.

Kamionkowski, M., Kosowsky, A., \& Stebbins, A., 1997, Phys. Rev. D, 55, 7368.

Keating, B.G., O'Dell, C.W., de Oliveira-Costa, A., Klawikowski, S., Stebor, N., Piccirillo, L., Tegmark, M., \& Timbie, P.T., 2001, ApJ, 560, L1.

Kesteven M., et al., 2002, in: Astrophysical Polarized Backgrounds, Cecchini, S., Cortiglioni, S., Sault, R., \& Sbarra, C. (eds.), AIP Conf. Proc., $609,156$.

Liu, G., Sugiyama, N., Benson, A.J., Lacey, C.G., \& Nusser, A., astro-ph/0101368.

Masi, S., et al., 2002, in: Astrophysical Polarized Backgrounds, Cecchini, S., Cortiglioni, S., Sault, R., \& Sbarra, C. (eds.), AIP Conf. Proc., $609,122$.
McClure-Griffiths, N.M., Green, A.J., Dickey, J.M., Gaensler, B.M., Haynes, R.F., \& Wieringa, M.H., 2001, ApJ, 551, 394.

Platania, P., Bensadoun, M., Bersanelli, M., De Amici, G., Kogut, A., Levin, S., Maino, D., \& Smoot, G.F., 1998, ApJ, 505, 473.

Seljak, U., \& Zaldarriaga, M., 1996, ApJ, 469, 437.

Seljak, U., \& Zaldarriaga, M., 1997, Phys. Rev. Lett., 78, 2054.

Seljak, U., 1997, ApJ, 482, 6.

Spoelstra, T.A.T., 1984, A\&A, 135, 238.

Staggs, S.T., Gundersen, J.O., \& Church, S.E., 1999, In: de Olivera-Costa A., Tegmark M. (eds.) Microwave Foregrounds, ASP Conf. Ser. Vol. 181, ASP, San Francisco, p. 299.

Tegmark, M., \& Efstathiou, G., 1996, MNRAS, $281,1297$.

Tegmark, M., Eisenstein, D.J., Hu, W., \& de Olivera-Costa, A., 2000, ApJ, 530, 133.

Toffolatti, L., Argüeso Gomez, F., De Zotti, G., Mazzei, P., Franceschini, A., Danese, L., \& Burigana, C., 1998, MNRAS, 297, 117.

Tucci, M., Carretti, E., Cecchini, S., Fabbri, R., Orsini, M., \& Pierpaoli, E., 2000, NewA, 5, 181.

Tucci, M., Carretti, E., Cecchini, S., Cortiglioni, S., Fabbri, R., \& Pierpaoli, E., 2001, Proc. 20th Texas Symposium on Relativistic Astrophysics, Craig, J.C., \& Martel, H. (eds), AIP Conf Proc. $586,184$.

Tucci, M., Carretti, E., Cecchini, S., Nicastro, L., Fabbri, R., Gaensler, B.M., Dickey, J.M., \& McClure-Griffiths, 2002, in: Astrophysical Polarized Backgrounds, Cecchini, S., Cortiglioni, S., Sault, R., \& Sbarra, C. (eds.), AIP Conf. Proc., 609, 60.

Uyaniker, B., et al., 1999, A\&AS, 138, 31.

Zaldarriaga, M., \& Seljak, U., 1997, Phys. Rev. D, $55,1830$.

Zaldarriaga, M., 2001, astro-ph/0106174. 
Zannoni, M., et al., 2002, in: Astrophysical Polarized Backgrounds, Cecchini, S., Cortiglioni, S., Sault, R., \& Sbarra, C. (eds.), AIP Conf. Proc., 609,115 .

Wieringa, M.H., de Bruyn, A.G., Jansen, D., Brouw, W.N., \& Katgert, P., 1993, A\&A, 268, 215.

Wright, E.L., 1999, NewAR, 43, 257; and MAP home page: http://map.gsfc.nasa.gov.

Fig. 1.- Linearly polarized intensity, $P I=$ $\sqrt{Q^{2}+U^{2}}$, in the Test Region. The image is obtained combining all nine spectral channels. The intensity scale runs from 0.4 to $9.5 \mathrm{mJy}^{\text {beam }^{-1}}$. The square boxes indicate the areas where we computed the angular power spectra.

This 2-column preprint was prepared with the AAS LATEX macros v5.0. 
Table 1: Best-fit parameters for angular power spectra

\begin{tabular}{ccccccccc}
\hline Survey $^{\dagger}$ & $\begin{array}{c}\text { Box } \\
(\mathrm{deg})\end{array}$ & $\begin{array}{c}\text { Centre } \\
(\mathrm{l}, \mathrm{b})\end{array}$ & $\begin{array}{c}A_{E} \\
\left(\mathrm{~K}^{2}\right)\end{array}$ & $\alpha_{E}$ & $\alpha_{B}$ & $\begin{array}{c}A_{P I} \\
\left(\mathrm{~K}^{2}\right)\end{array}$ & $\alpha_{P I}$ & $\begin{array}{c}\ell-\text { range } \\
\left(\times 10^{3}\right)\end{array}$ \\
\hline $\mathrm{D} 97$ & $5 \times 5$ & $(329,1.5)$ & $9.1 \times 10^{-4}$ & $1.78 \pm 0.18$ & $1.62 \pm 0.19$ & $3.6 \times 10^{-4}$ & $1.68 \pm 0.30$ & $(0.1,0.8)$ \\
\hline $\mathrm{G} 01$ & $4 \times 4$ & $(329,1.5)$ & 93.1 & $2.85 \pm 0.07$ & $2.74 \pm 0.06$ & $4.2 \times 10^{-3}$ & $1.66 \pm 0.07$ & $(0.6,6)$ \\
& $3 \times 3$ & $(330.5,1.5)$ & 60.2 & $2.76 \pm 0.09$ & $2.65 \pm 0.10$ & $6.0 \times 10^{-3}$ & $1.67 \pm 0.08$ & $(0.6,6)$ \\
& $3 \times 3$ & $(327.5,1.5)$ & 63.0 & $2.84 \pm 0.09$ & $2.70 \pm 0.10$ & $3.7 \times 10^{-3}$ & $1.68 \pm 0.08$ & $(0.6,6)$ \\
\hline
\end{tabular}

$\dagger$ For the D97 survey the $A_{X}$ values are at $2.4 \mathrm{GHz}$, for the Test Region (G01) at $1.4 \mathrm{GHz}$. In the last column we report the range of angular scales considered in the fit.

Table 2: The best-fit parameters for $1^{\circ} \times 1^{\circ}$ areas in the $\ell$-range $[1000,6000]$

\begin{tabular}{cccccccc}
\hline Box Label $^{\dagger}$ & $\begin{array}{c}\text { Box Centre } \\
(l, b)\end{array}$ & $\begin{array}{c}P I_{\text {rms }} \\
(\mathrm{K})\end{array}$ & $\begin{array}{c}A_{E} \\
\left(\mathrm{~K}^{2}\right)\end{array}$ & $\alpha_{E}$ & $\alpha_{B}$ & $\begin{array}{c}A_{P I} \\
\left(\mathrm{~K}^{2}\right)\end{array}$ & $\alpha_{P I}$ \\
\hline $\mathrm{a}$ & $(330.6,1.0)$ & 0.46 & 168 & $2.84 \pm 0.25$ & $2.58 \pm 0.24$ & $2.1 \times 10^{-2}$ & $1.79 \pm 0.20$ \\
$\mathrm{~b}$ & $(329.7,1.1)$ & 0.54 & $2.9 \times 10^{3}$ & $3.18 \pm 0.26$ & $3.24 \pm 0.30$ & 0.89 & $2.24 \pm 0.22$ \\
$\mathrm{c}$ & $(327.7,1.1)$ & 0.38 & 137 & $2.91 \pm 0.30$ & $2.97 \pm 0.28$ & $3.4 \times 10^{-2}$ & $1.93 \pm 0.27$ \\
$\mathrm{~d}$ & $(328.5,2.6)$ & 0.38 & $1.8 \times 10^{5}$ & $3.84 \pm 0.34$ & $3.53 \pm 0.35$ & 5.1 & $2.58 \pm 0.29$ \\
$\mathrm{e}$ & $(327.0,2.3)$ & 0.26 & 616 & $3.18 \pm 0.35$ & $3.07 \pm 0.32$ & $1.0 \times 10^{-2}$ & $1.88 \pm 0.38$ \\
$\mathrm{f}$ & $(330.4,2.3)$ & 0.31 & 233 & $3.01 \pm 0.38$ & $2.64 \pm 0.39$ & $4.4 \times 10^{-3}$ & $1.68 \pm 0.35$ \\
\hline
\end{tabular}

$\dagger$ The letters correspond to the panels in Fig. 6. 


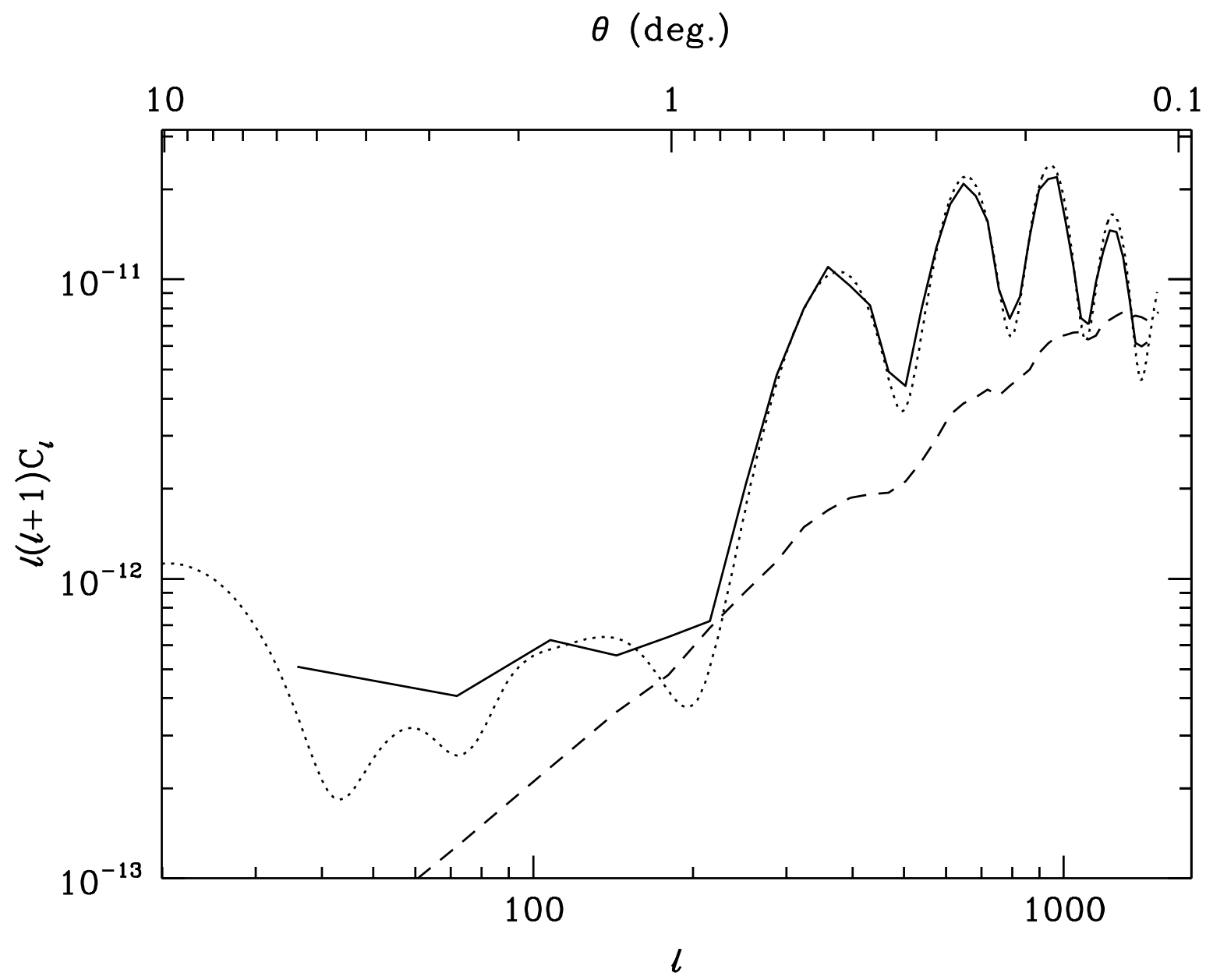

Fig. 2.- The CMB spectra for $E$-mode (dotted line is the input spectrum, the solid line is the computed spectrum from Fourier analysis) and for the PI field (dashed line), from a CDM model with a secondary ionization optical depth $\tau_{i o n}=0.2$. 


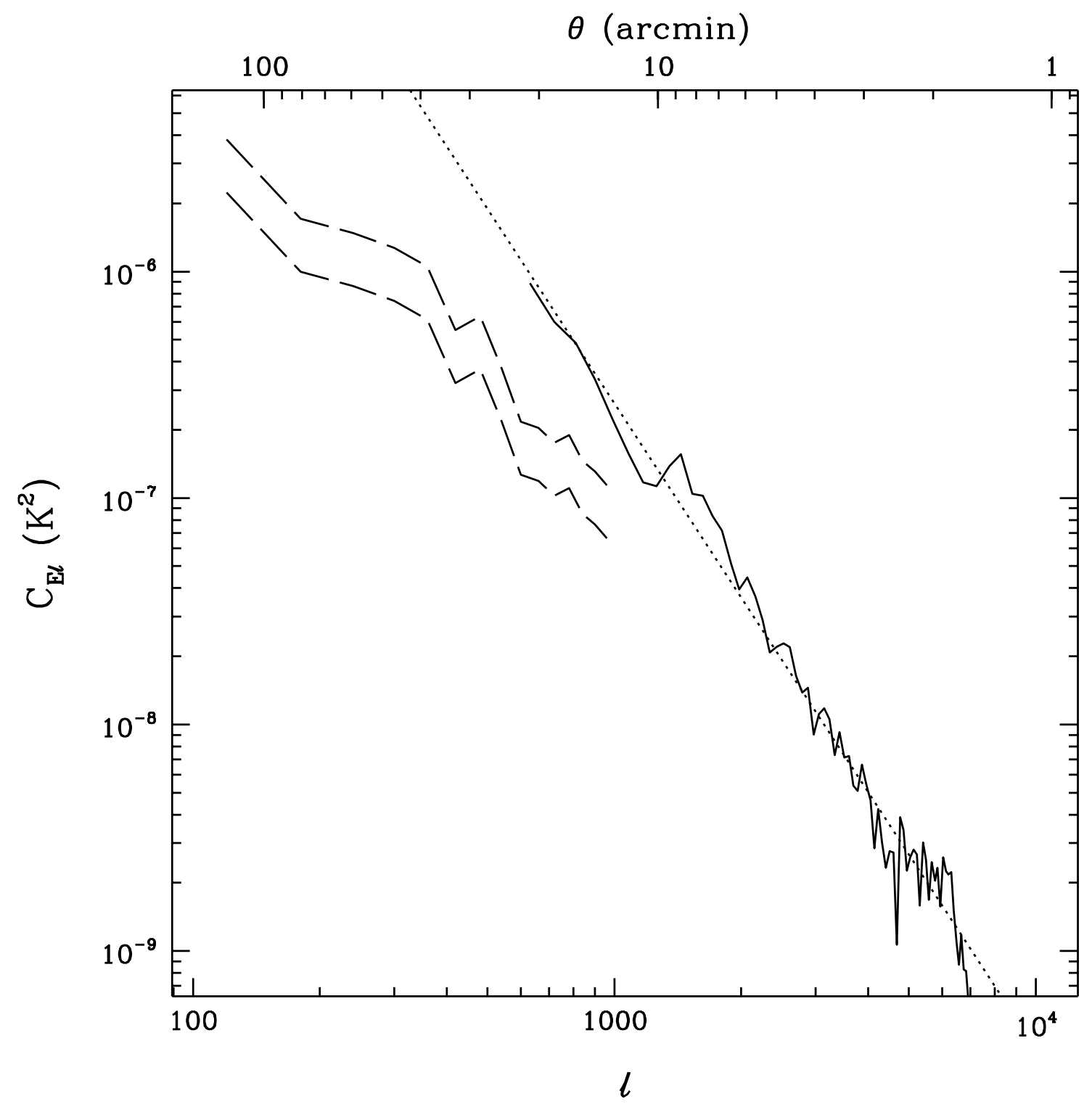

Fig. 3. - E-mode spectrum from the $4^{\circ} \times 4^{\circ}$ region (solid lines), compared to the result from D97 data (dashed lines) in a $5^{\circ} \times 5^{\circ}$ area centered on the same position. The dotted line is the best-fit power law in the $\ell$-range between 600 and 6000 (see Table 1 ). 


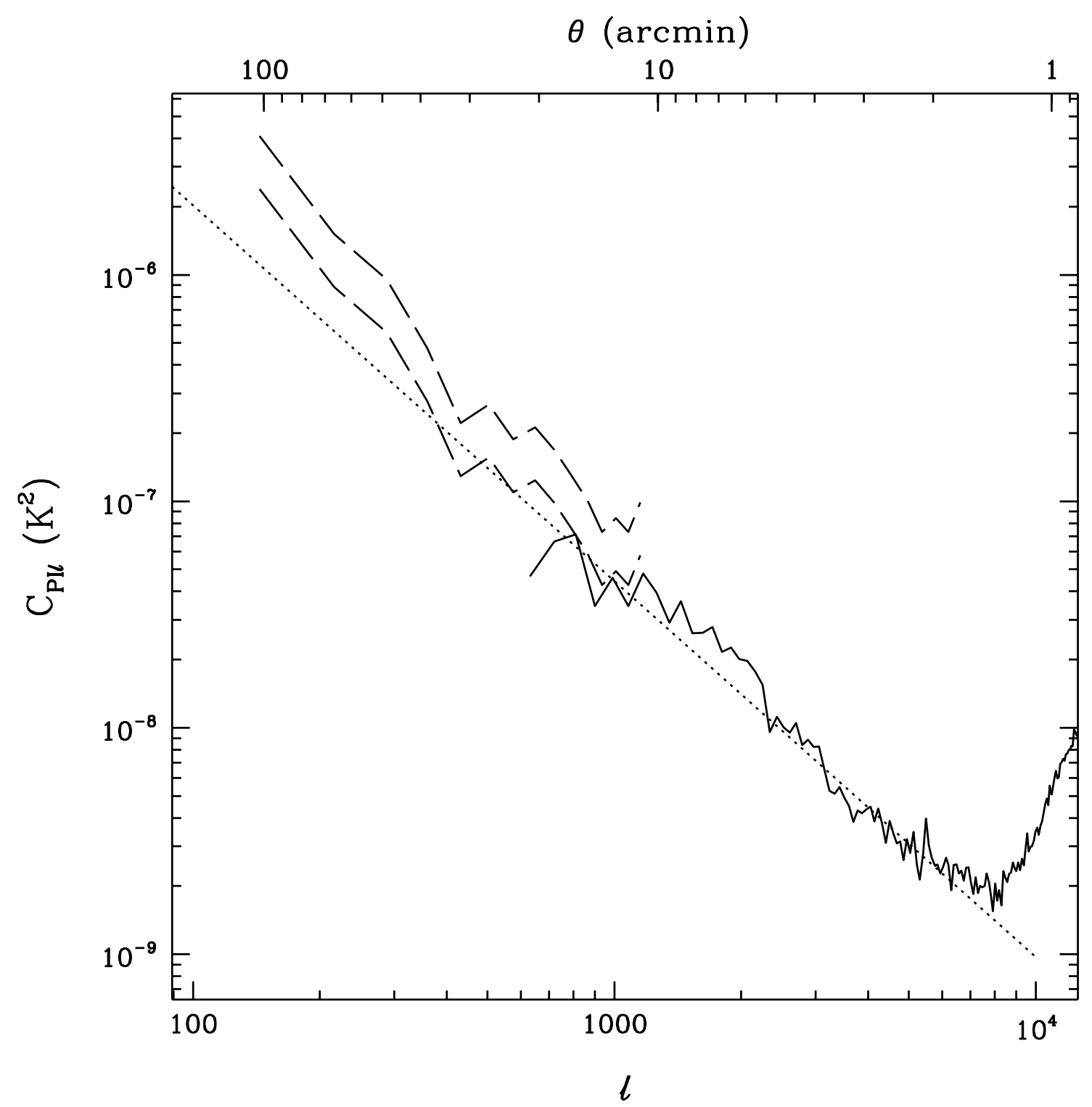

Fig. 4.- As in Fig. 3, but for polarization intensity spectra. 

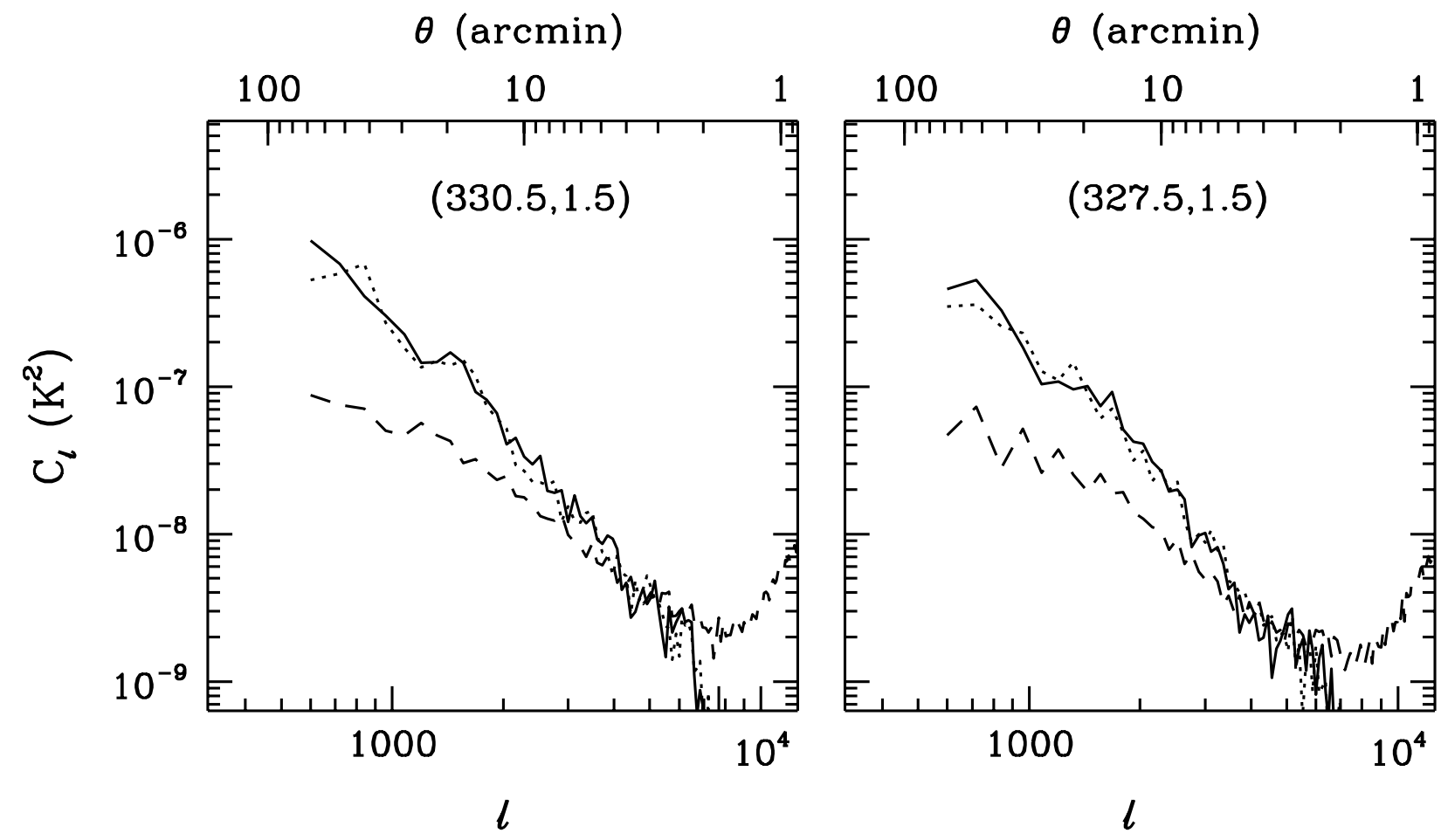

Fig. 5. $-C_{E \ell}$ (solid lines), $C_{B \ell}$ (dotted lines) and $C_{P I \ell}$ (dashed lines) in two $3^{\circ} \times 3^{\circ}$ boxes, whose centers are shown in the panels. 

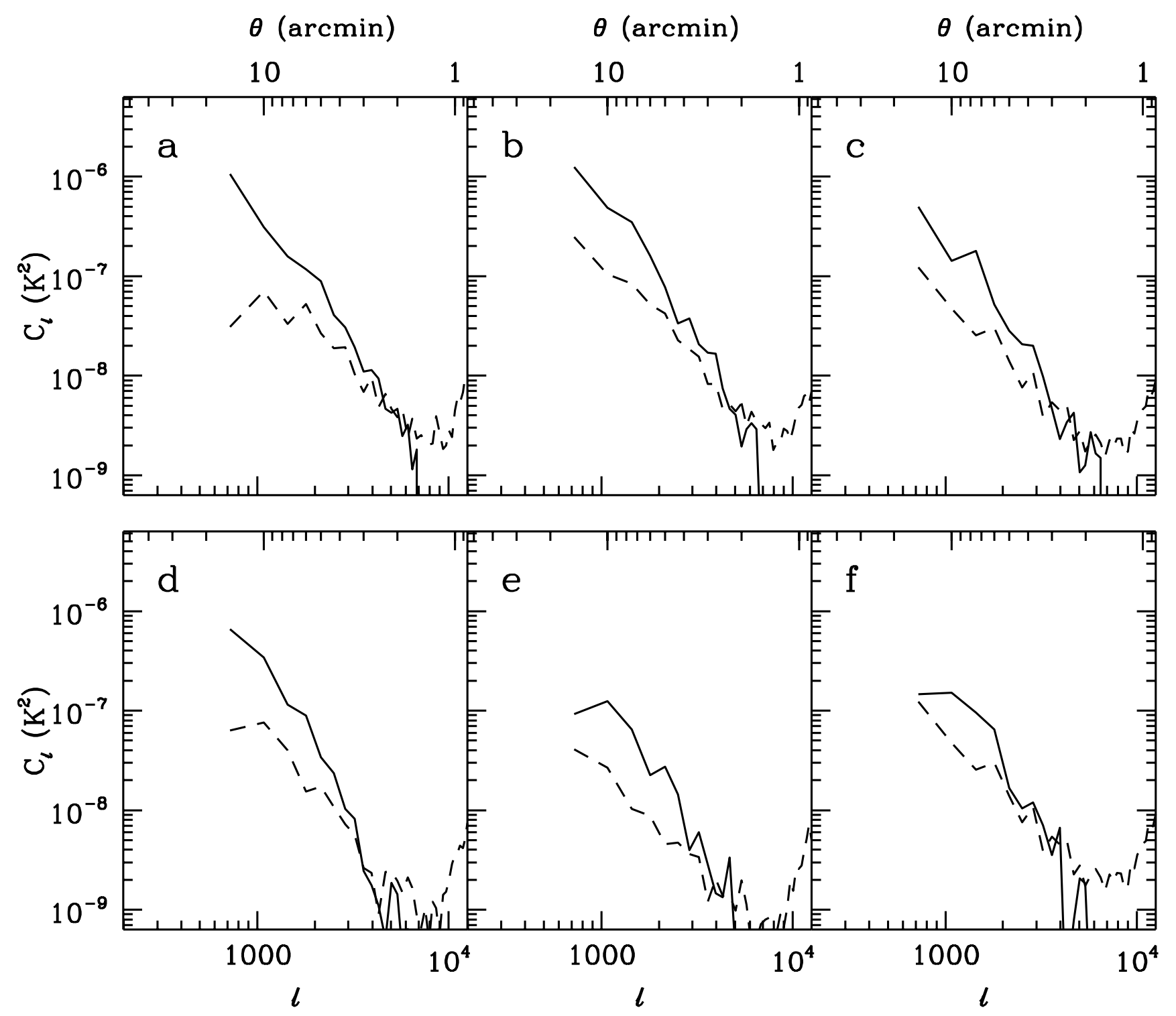

Fig. 6. $-C_{E \ell}$ (solid lines) and $C_{P I \ell}$ (dashed lines) in six $1^{\circ} \times 1^{\circ}$ boxes. The labels in the plots identify the regions where the spectra are computed. 
This figure "f1.jpg" is available in "jpg" format from: http://arxiv.org/ps/astro-ph/0207237v1 